\title{
Thromboprophylaxis decreases the risk of pulmonary embolism in COVID-19
}

\author{
๑D Murat Uğur ${ }^{1}$, @Taha Yusuf Kuzan², @Nurettin Yiyit ${ }^{3}$ \\ ${ }^{1}$ Health Sciences University, Sancaktepe Şehit Prof. Dr. İlhan Varank Education and Research Hospital, Department of Cardiovascular Surgery, \\ İstanbul, Turkey \\ ${ }^{2}$ Health Sciences University, Sancaktepe Şehit Prof. Dr. İlhan Varank Education and Research Hospital, Department of Radiology, İstanbul, Turkey \\ ${ }^{3}$ Health Sciences University, Sancaktepe Şehit Prof. Dr. İlhan Varank Education and Research Hospital, Department of Thoracic Surgery, İstanbul, \\ Turkey
}

Cite this article as: Uğur M, Kuzan TY, Yiyit N. Thromboprophylaxis decreases the risk of pulmonary embolism in COVID-19. Anatolian Curr Med J 2021; 3(3); 188-191.

\begin{abstract}
Aim: Pulmonary embolism caused by micromboli and microthrombuses might be associated with increased mortality and morbidity in COVID-19. Routine thromboprophylaxis application might contribute to clinical recovery by reducing the risk of pulmonary embolism. In this study, the effects of thromboprophylaxis on clinical outcomes were investigated by examining the rates of computed tomographic angiography requests for the differential diagnosis of pulmonary embolism in the patients with COVID-19.

Material and Method: Patients who underwent computed tomography (CT) between 10 March and 25 May 2020 in our hospital were retrospectively analysed. Patients who received simultaneous PCR tests during CT examination were included in the study. In the follow-up of these patients, rates of pulmonary CT angiography request with the suspicion of pulmonary embolism and it's were investigated.

Results: During the study period, thorax CT was performed in 11142 patients and pulmonary CT angiography was performed in $161(1.4 \%)$ of them. Ninety-nine patients who were performed CT angiography and PCR test during the hospitalization period were included in the study. PCR test was positive in $22 \%(\mathrm{n}=21)$ of the patients $(22 \%)$ and pulmonary embolism was detected in 6 of them (6.3\%). PCR test was negative in 74 patients (78\%) and $17(17.9 \%)$ had pulmonary embolism.

Conclusion: Routine thromboprophylaxis contributes to the treatment of COVID-19 by preventing the development of microthrombosis and thrombotic complications in the respiratory system.
\end{abstract}

Keywords: COVID-19, Coronavirus, pulmonary embolism, thrombosis

\section{INTRODUCTION}

COVID-19, originating from China, has been declared a pandemic in the short term and has become a disease that threatens the whole world and changes life habits. Tendency to thrombosis increases in the etiopathogenesis of the disease. Abnormal blood clotting and thrombosis have been reported as a predictive factor for the poor prognosis (1). In COVID-19, similar to SARS CoV, thrombosis might develop in small and medium-sized pulmonary arteries (2). The disease is characterized by pulmonary involvement and pulmonary embolism might worsen the clinical course $(2,3)$. Pulmonary embolism was detected in $23-37 \%$ of critically ill patients with COVID-19 $(4,5)$.
In Turkey, according to the treatment algorithm, determined by the Ministry of Health, it was aimed to avoid the complications related to thrombosis by prophylaxis with low molecular weight heparin to every hospitalized patient, besides hydroxychloroquine (6). Pulmonary computed tomography (CT) angiography is a diagnostic imaging method for the diagnosis of pulmonary embolism. It is the first applied diagnostic method to confirm the diagnosis in case of doubt. In this study, number of pulmonary CT angiography and rates of pulmonary embolism were evaluated in our hospital, which is a pandemic hospital in the fight against COVID-19. 


\section{MATERIAL AND METHOD}

Patients who underwent computed tomography (CT) between 10 March-25 May 2020 in our hospital were evaluated retrospectively. Patients older than 18 years old and who underwent PCR testing for COVID-19 concomitant to chest CT were included in the study. Patients under the age of eighteen and not performed PCR testing were excluded from the study. Patients, who were performed thorax CT, were determined from the patients' files. Pulmonary CT angiography examination rates with the suspicion of pulmonary embolism were evaluated. The study was carried out with the permission of Scientific Researches Ethics Committee of Sancaktepe Sehit Prof. Dr. İlhan Varank Education and Research Hospital (Date: 19.09.2020, Decision No: 2020/1). All procedures were performed adhered to the ethical rules, and principles of the Helsinki Declaration.

Samples were taken for PCR test from all patients, who were admitted to our hospital with the suspicion of COVID-19, during the pandemic period. According to the our hospital criteria, thorax CT was performed in the patients with signs of pulmonary involvement or all hospitalized patients due to its high sensitivity and low specificity in the diagnosis of COVID-19 (7). Since thorax CT was not planned without PCR testing from any COVID-19 suspected patient, patients who underwent thorax CT without PCR testing were excluded from the study.

Patients with the diagnosis of COVID-19 were treated according to the treatment algorithms of the Ministry of Health (6). While hydroxychloroquine treatment was started routinely in all patients, low molecular weight heparin was added to the routine treatment since April 12. Before this date, prophylactic thromboprophylaxis was applied to immobile patients, patients with moderate-tosevere respiratory failure and patients necessitating intensive care unit. In patients with severe respiratory distress, low molecular heparin was administered with treatment dose. The data were analyzed in Microsoft Office Excel.

\section{RESULTS}

In our hospital, 11,142 thorax CT was performed between March 10 and May 25, 2020. Pulmonary CT angiography was performed in 161 (1.4\%) of these patients. PCR test was not requested in 62 of the patients underwent pulmonary CT angiography were not suspected of Covit-19. Pulmonary CT angiography was done in 99 patients with PCR tests due to suspicion of pulmonary embolism. Four of the pulmonary CT angiographies were non-diagnostic. PCR test was positive in 21 (22\%) of the remaining 95 patients, and pulmonary embolism was detected in $6(6.3 \%)$ of them (Figure 1-2). PCR was negative in 74 (78\%) of which 17 (17.9\%) had pulmonary embolism.

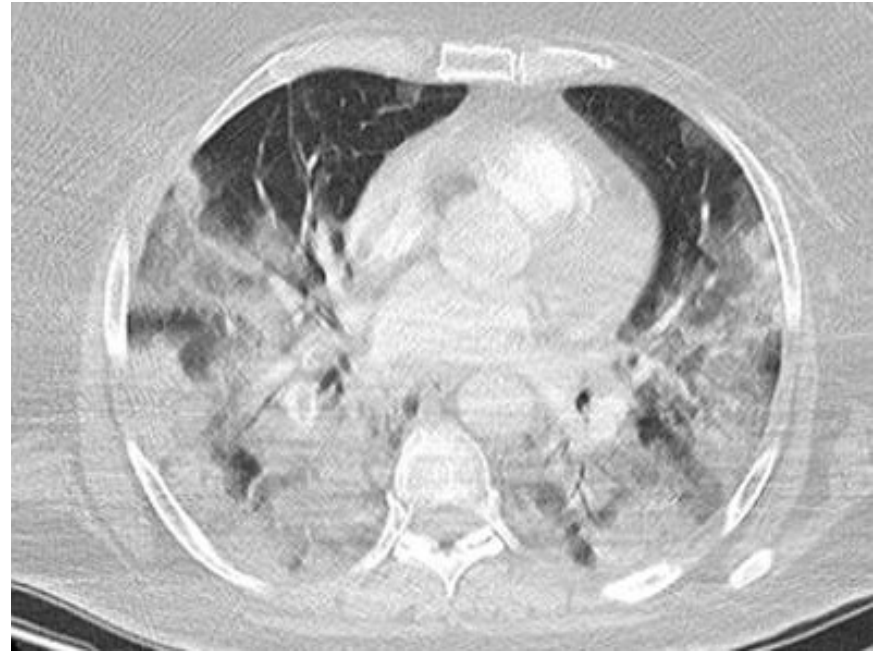

Figure 1. Central and peripheral ground glass opacities and consolidation compatible with COVID-19 pneumonia in thorax CT.

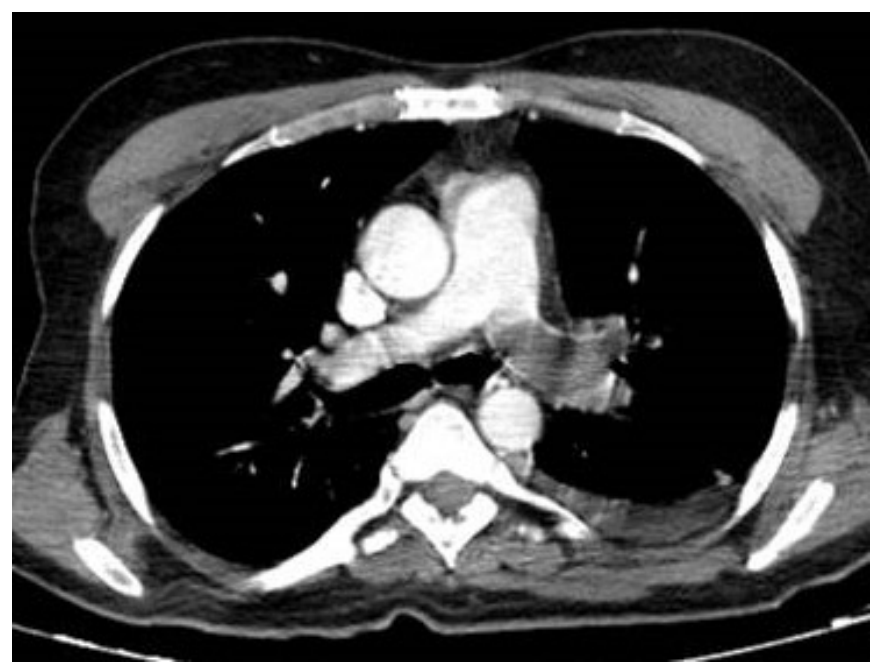

Figure 2. Pulmonary CT angiography of massive pulmonary thrombus in left main pulmonary artery.

Table 1. Results of the pulmonary CT angiography in the hospitalized patients with the diagnosis of COVID-19.

\begin{tabular}{|lccc|}
\hline & $\begin{array}{c}\text { Positive } \\
\text { Pulmonary } \\
\text { Embolism } \\
\text { in CT } \\
\text { Angiography }\end{array}$ & $\begin{array}{c}\text { Negative } \\
\text { Pulmonary } \\
\text { Embolism in CT } \\
\text { Angiography }\end{array}$ & Total \\
\hline PCR positive & 6 & 15 & 21 \\
CT undefined & 2 & 5 & 7 \\
$\begin{array}{l}\text { CT compatible } \\
\text { for COVID-19 }\end{array}$ & 3 & 7 & 10 \\
$\begin{array}{l}\text { CT incompatible } \\
\text { for COVID-19 }\end{array}$ & 1 & 3 & 4 \\
PCR negative & 17 & 57 & 74 \\
\hline $\begin{array}{l}\text { CT undefined } \\
\text { CT compatible } \\
\text { for COVID-19 }\end{array}$ & 3 & 11 & 14 \\
\hline $\begin{array}{l}\text { CT incompatible } \\
\text { for COVID-19 }\end{array}$ & 4 & 7 & 17 \\
Total & 23 & 39 & 43 \\
\hline CT: Computerized tomography & & 72 & 95 \\
\hline
\end{tabular}




\section{DISCUSSION}

Routine thrombosis prophylaxis in the treatment of COVID-19 contributes to the clinical course by preventing the development of micro-emboli in the patients. In our hospital, thromboprophylaxis was applied to the patients with COVID-19 depending on the algorithms of the Ministry of Health (6). As a result of routine prophylaxis, pulmonary CT angiography request was found to be quite low due to the suspicion of pulmonary embolism.

COVID-19 might cause arterial and venous thrombosis directly or by causing endothelial damage due to infection, inflammation, immobilization and hypoxia (2-8). The coronovirus might affect many organs, including the lung, heart, vascular system, kidney and intestine through the angiotensin converting enzyme 2 (ACE-2) receptor $(2,6,11)$. COVID-19 activates the renin-angiotensin system (RAS) by binding to ACE-2 receptors. Thus, it increases the tendency to thrombosis by increasing platelet adhesion and aggregation (9). Also, inflammatory cytokines secreted due to the disease might activate the coagulation cascade, causing thrombosis (1). Cytokine storm might play an important role in initiating and promoting thrombosis in arteries and veins (12). Cytokine storm in sepsis might damage monocytes and endothelial cells, causing disseminated intravascular coagulation (DIC) (13). Prevention of cytokine storm might reduce the risk of thrombosis in patients with COVID-19 and alleviate the progression of the disease (12). Thromboprophylaxis with low molecular heparin decrease the risk of embolic events with its anticoagulant effect and might help to decrease the risk of cytokine storm with its anti-inflammatory effect (14).

Acute respiratory distress syndrome (ARDS) is not the only pathology in pulmonary involvement of COVID-19, microvascular thrombosis also plays a role in pathogenesis (2). Complement accumulation in the vascular bed, which is an important feature of many micro thrombotic syndromes such as antiphospholipid antibody syndrome, atypical hemolytic uremic syndrome, purpura fulminant, was also reported in COVID-19. Complement accumulation might be accompanied by septal capillary injury in COVID-19 (14).

Hypercoagulability develops and D-dimer increases in the COVID-19 patients with progressed respiratory infection. Prothrombin time (PT) becomes longer and platelet level decreases as the disease becomes serious. Free thrombin, which is not controlled by natural anticoagulants in circulation, activates platelets and stimulates fibrinolysis. Accordingly, levels of D-Dimer and fibrin degradation products (FDP) increase (13). D-dimer and FDP levels were found significantly elevated in patients who were lost, suggesting a common coagulation activation, irregular thrombin production, impaired natural anticoagulants and fibrinolysis might be effective in the pathogenesis of the disease (15). Early recognition of thrombosis or routine prophylaxis will contribute to improve the clinical outcome of the patients. Thromboprophylaxis in COVID-19 patients is recommended for all hospitalized patients in Turkey country since April with recommendation of scientific committee of Ministry of Health (6). Routine prophylaxis decreases the thrombogenic effect of the virus by preventing deep venous thrombosis and related complications due to immobilization along with microemboli that might develop in the pulmonary system.

In the standard approach to the hospitalized patients, anticoagulant therapy is not routinely recommended for mobile patients. Patients with COVID-19 are at risk for venous thrombosis due to the severity of the infection and immobilization. Risk of venous thrombosis in hospitalized cases with COVID-19 was reported as $40 \%(8,17)$. It has been reported that venous thrombosis was developed in $25 \%$ of ICU patients without prophylaxis (1). In the evaluation of 184 patients with COVID-19, thrombosis was detected in $1 / 3$ of intensive care patients despite standard prophylaxis, and segmental or subsegmental pulmonary embolism was found in $80 \%$ of venous thrombosis (3).

Anticoagulation to be started early term in COVID-19 patients contributes to prognosis by reducing the risk of micro-thrombus. $(11,13)$. It is suggested that to apply prophylactic anticoagulants to all hospitalized patients with the diagnosis of COVID-19, to perform CT angiography in the patients with suspicion of pulmonary embolism, and to use therapeutic dose of LMWH if CT examination was not possible (2). Thrombophylaxis was applied only to immobile patients, as in other diseases, at the beginning of the fight against COVID-19. Upon the determination of the microthrombosis and microemboli caused by the virus, thromboprophylaxis is recommended to all hospitalized patients in our country since April (6). In Turkey, in the comparison with other European countries, earlier reduction of intensive care unit need, number of hospitalization and COVID-19 patients, might be associated with starting LMWH in all hospitalized patients.

Dependingontherisk oftransmission and general condition of the patient, the examinations tests to be performed in COVID-19 patients may be restricted. Changes of D-dimer levels might be caused by hypercoagulability or severity of the disease (9). Since the D-dimer levels, which is used for differential diagnosis of thrombosis, might be increased by direct effect of COVID-19, it cannot be used to diagnosis of thrombosis in COVID-19. Therefore, pulmonary CT angiography is appropriate for clinical suspicion of pulmonary embolism. Increasing the anticoagulant dose to the therapeutic dose might prevent progression of the disease in the patients with moderate and severe symptoms. As a result of routine prophylaxis 
in our hospital, only $1.4 \%$ of our patients required CT angiography with suspicion of pulmonary embolism and only $24 \%$ of these patients had pulmonary embolism. Considering that CT angiography was performed only in patients with highly suspicion of pulmonary embolism in order to decrease the risk of transmission during the pandemic period, this rate might be considered as an indication that thromboprophylaxis significantly reduces the risk of pulmonary embolism in COVID-19.

Limitation of the study is data loss due to the nature of the retrospective study. Although LMWH treatment has been routine since April, it was not known how many patients who have undergone CT angiography with the suspicion of pulmonary embolism were used prophylaxis. The cases with pulmonary embolism and severe COVID-19 infection, who were admitted to the emergency department in serious condition and directly transferred to the intensive care unit, might not be diagnosed. However, in the evaluation of the need and results of CT angiography rates, it might be considered that routine prophylaxis with LMWH reduces the clinical suspicion of pulmonary embolism in COVID-19.

\section{CONCLUSION}

COVID-19 is a disease, characterized by respiratory system involvement, and severity of the disease is associated with microthrombosis and microembolism in pulmonary artery or other vascular beds. Adding thromboprophylaxis to the treatment of the COVID-19 contributes positively to the clinical course by reducing the risk of venous thrombosis, which might cause pulmonary embolism, and thrombosis that might develop in the pulmonary arteries.

\section{ETHICAL DECLARATIONS}

Ethics Committee Approval: The study was carried out with the permission of Scientific Researches Ethics Committee of Sancaktepe Sehit Prof. Dr. İlhan Varank Education and Research Hospital (Date: 19.09.2020, Decision No: 2020/1).

Informed Consent: All patients signed the free and informed consent form.

Referee Evaluation Process: Externally peer-reviewed.

Conflict of Interest Statement: The authors have no conflicts of interest to declare.

Financial Disclosure: The authors declared that this study has received no financial support.

Author Contributions: All of the authors declare that they have all participated in the design, execution, and analysis of the paper, and that they have approved the final version.

\section{REFERENCES}

1. Cui S, Chen S, Li X, Liu S, Wang F. Prevalence of venous thromboembolism in patients with severe novel coronavirus pneumonia. J Thromb Haemost 2020; 18: 1421-4.

2. Oudkerk M, Büller HR, Kuijpers D, et al. Diagnosis, Prevention, and Treatment of Thromboembolic Complications in COVID-19: Report of the National Institute for Public Health of the Netherlands. Radiology 2020; 297: 216-22.

3. Klok FA, Kruip MJHA, van der Meer NJM, et al. Incidence of thrombotic complications in critically ill ICU patients with COVID-19. Thromb Res 2020; 191: 145-7.

4. Grillet F, Behr J, Calame P, Aubry S, Delabrousse E. Acute pulmonary embolism associated with COVID-19 Pneumonia Detected with Pulmonary CT Angiography. Radiology 2020; 296: E186-E188

5. Whyte MB, Kelly PA, Gonzalez E, Arya R, Roberts LN. Pulmonary embolism in hospitalised patients with COVID-19. Thromb Res 2020; 195: 95-99.

6. COVID-19 (SARS-CoV-2 infection) guide. Science Committee Work. Ministriy of Health, Public Health General Directorate. Ankara, April, 2020.

7. Kuzan TY, Murzoğlu Altıntoprak K, Çiftçi HÖ, et al. A comparison of clinical, laboratory and chest CT findings of laboratoryconfirmed and clinically diagnosed COVID-19 patients at first admission. Diagn Interv Radiol. 2020; 10.5152/dir.2020.20270.

8. Bikdeli B, Madhavan MV, Jimenez D, et al. COVID-19 and thrombotic or thromboembolic disease: implications for prevention, antithrombotic therapy, and follow-up: JACC State-ofthe-Art review. J Am Coll Cardiol 2020; 75: 2950-73.

9. Liu X, Li Z, Liu S, et al. Potential therapeutic effects of dipyridamole in the severely ill patients with COVID-19. Acta Pharm Sin B 2020; 10: 1205-15.

10. Zhang H, Penninger JM, Li Y, Zhong N, Slutsky AS. Angiotensinconverting enzyme 2 (ACE2) as a SARS-CoV-2 receptor: Molecular mechanisms and potential therapeutic target. Intensive Care Med 2020; 46: 586-90.

11. Li T, Lu H, Zhang W. Clinical observation and management of COVID-19 patients. Emerg Microbes Infect 2020; 9: 687-90.

12. Barnes BJ, Adrover JM, Baxter-Stoltzfus A, et al. Targeting potential drivers of COVID-19: Neutrophil extracellular traps. J Exp Med 2020; 217: e20200652.

13. Tang N, Li D, Wang X, Sun Z. Abnormal coagulation parameters are associated with poor prognosis in patients with novel coronavirus pneumonia. J Thromb Haemost 2020; 18: 844-7.

14. Miesbach W, Makris M. COVID-19: Coagulopathy, risk of thrombosis, and the rationale for anticoagulation. Clin Appl Thromb Hemost 2020; 26: 1076029620938149.

15. Magro C, Mulvey JJ, Berlin D, et al. Complement associated microvascular injury and thrombosis in the pathogenesis of severe COVID-19 infection: a report of five cases. Transl Res 2020; 220: $1-13$.

16. Frater JL, Zini G, d'Onofrio G, Rogers HJ. COVID-19 and the clinical hematology laboratory. Int J Lab Hematol. 2020; 42: 11-8.

17. Wang T, Chen R, Liu C, et al. Attention should be paid to venous thromboembolism prophylaxis in the management of COVID-19. Lancet Haematol 2020; 7: 362-3. 